\title{
End-effector design optimisation and multi-robot motion planning for handling compliant parts
}

\author{
Emile Glorieux $^{1}$ (D) Pasquale Franciosa ${ }^{2}$. Darek Ceglarek $^{2}$
}

Received: 9 March 2017 / Revised: 23 August 2017 / Accepted: 24 August 2017 / Published online: 9 September 2017

(C) The Author(s) 2017. This article is an open access publication

\begin{abstract}
The deformation of compliant parts during material handling is a critical issue that can significantly affect the productivity and the parts' dimensional quality. There are multiple relevant aspects to consider when designing end-effectors to handle compliant parts, e.g. motion planning, holding force, part deformations, collisions, etc. This paper focuses on multi-robot material handling systems where the end-effector designs influence the coordination of the robots to prevent that these collide in the shared workspace. A multi-disciplinary methodology for end-effector design optimisation and multi-robot motion planning for material handling of compliant parts is proposed. The novelty is the co-adaptive optimisation of the end-effectors' structure with the robot motion planning to obtain the highest productivity and to avoid excessive part deformations. Based on FEA, the dynamic deformations of the parts are modelled in order to consider these during the collision avoidance between the handled parts and obstacles. The proposed methodology is evaluated for a case study that considers the multi-robot material handling of sheet metal parts in a multi-stage tandem press line. The results show that a substantial improvement in productivity can be achieved (up to $1.9 \%$ ). These also demonstrate the need and contribution of the proposed methodology.
\end{abstract}

Emile Glorieux

emile.glorieux@hv.se

1 Department of Engineering Science, University West, S-461 86, Trollhättan, Sweden

2 Warwick Manufacturing Group, University of Warwick, CV4 7AL Coventry, UK
Keywords End-effector design optimisation · Motion planning $\cdot$ Multi-robot systems $\cdot$ Material handling · Compliant parts

\section{Introduction}

Compliant sheet metal parts are widely used in manufacturing industries such as automotive, aerospace, and appliance. The compliance of the parts makes them deform during the robotic material handling in manufacturing systems. Controlling those deformations is necessary to maintain the dimensional quality. The magnitude of the part deformations is influenced by robot motion planning parameters (i.e. transfer paths, trajectory velocities and accelerations) and design parameters (i.e. end-effector design). Hence, the effect of the part deformations can become a limitation for the productivity ( $\mathrm{Li}$ and Ceglarek 2002). In multi-robot systems, there is also the additional difficulty that several robots operate in close proximity. A typical example is the multi-robot material handling in multi-stage sheet metal press lines where the plates need to be transferred between the presses as quick as possible.

There are several issues that arise with multi-robot handling of compliant parts. A first issue is to tune the velocities and accelerations of the robots to avoid permanent plastic deformations of the parts during handling in order to maintain the dimensional quality and to avoid damaging the parts.

A second issue is to anticipate for all potential collisions between the deformed parts and obstacles in the workspace, which makes it necessary to consider the part deformations, instead of assuming it is rigid, during the collision detection simulation. For example with relatively large steel plates (e.g. $1000 \times 1500 \times 1 \mathrm{~mm}$ ), the allowed elastic 
deformation can be over 150mm. Fig. 1 shows this for the press line example. When assuming that the part is rigid (Fig. 1a), the collision between the deformed part and the lower die (Fig. 1b) remains undetected.

A third issue is that even elastic part deformations during handling can affect the dimensional quality (Li et al. 2002), for example due to position errors or part distortions, and cause large dimensional variations and/or parts that have to be scrapped. The larger the magnitude of the part deformations, the higher the risk that this happens. In the sheet metal press line example, large elastic deformations increase nesting errors ( $\mathrm{Li}$ et al. 2002), which are positioning errors of the plate in the lower die when it is dropped by the endeffector. This causes dimensional variations when the plate is stamped. Another problem is the part distortions during die contact ( $\mathrm{Li}$ et al. 2002), which occur when the endeffector drops a plate in the die and the contact force is unevenly distributed due to the deformations.

A fourth issue is that the design of the end-effectors not only affects the part deformations but also plays a crucial role in avoiding collisions between the robots (and other moving obstacles). The end-effectors are often the first to interfere with the other robot(s) in the case of a collision. Modifying the design of the end-effectors will affect to what extend the robots are able to operate simultaneously in the shared workspace. Hence, the end-effector designs

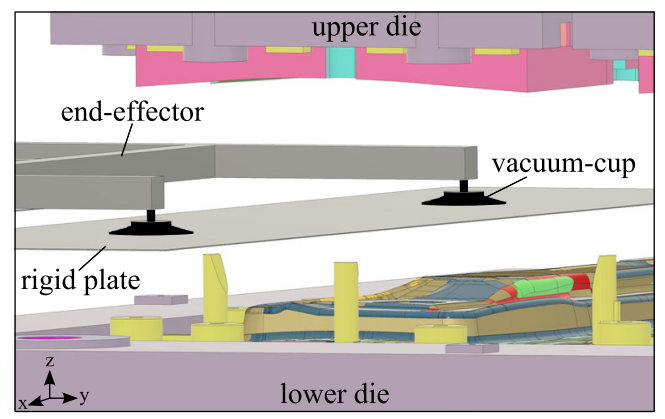

(a)

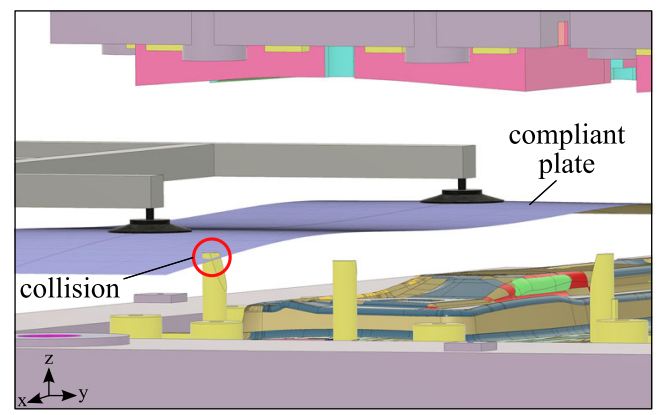

(b)

Fig. 1 Loading blank plate into press (a) rigid plate model, b compliant plate model (collision between plate and lower die indicated by red circle) influence the multi-robot coordination and consequently the productivity. Figure 2 illustrates this for the sheet metal press line example, looking at how long the press needs to wait until the end-effector that loaded the unstamped has retracted (to the left) before the upper die of the press can move downwards to perform the stamping operation. It can be seen that the design of the end-effector influences the waiting time before starting the stamping operation by the press. The position of the robot is the same in both scenarios, but the end-effector in Fig. 2a is larger compared to Fig. 2b. It shows that the position of the press is higher in Fig. 2a because it has to wait longer for the larger endeffector to retract from the shared workspace. This will result in a longer cycle-time compared to scenario with the smaller end-effector shown in Fig. $2 b$.

Designing the end-effectors and planning multiple robot motions are thus two inter-dependent problems that together determine the system's productivity. The topic of this paper is how to co-adaptively optimise these two problems to account for this inter-dependency.

The contribution of this paper is the proposed multidisciplinary methodology to simultaneously optimise the end-effectors' design and the manipulation planning in multi-robot material handling systems to exploit the synergies to improve the productivity. The novelty of the proposed methodology is that it integrates the end-effectors'

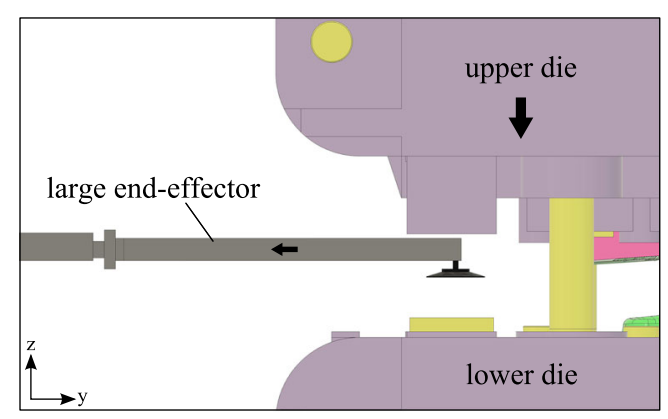

(a)

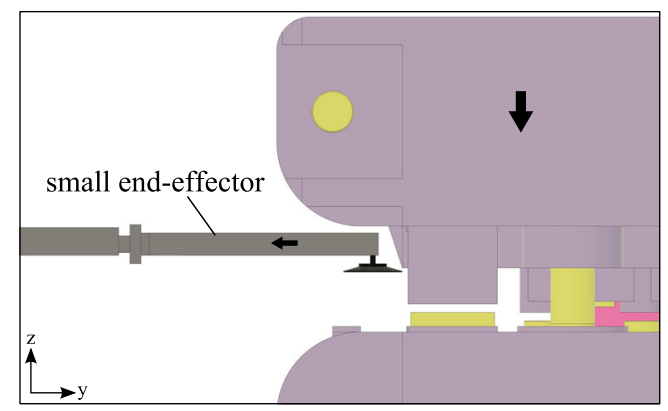

(b)

Fig. 2 Retracting end-effector after loading late into press, a large end-effector $\mathbf{b}$ small end-effector 
design problem with the main relevant aspects of robot motion planning. The methodology thereby co-adapts these aspects to each other in order to further improve the productivity of the system. The industrial application is presented in a case study that considers the material handling in a real-world tandem sheet metal press line for multi-stage stamping.

The remainder of the paper is outlined as follows, Section 2 gives an overview of related research works, followed by Section 3 that presents the considered case study. Section 4 formulates the investigated problem and discusses how it is modelled. Next, the proposed optimisation methodology is presented in detail in Section 5. Section 6 describes how the proposed methodology is implemented for the case study. The performed tests for the evaluation are presented in Section 7 and the results are discussed in Section 8. Finally, a summary and the conclusions are discussed in Section 9.

\section{Relevant works}

Two categories of research are relevant for this paper, firstly research on design optimisation for material handling end-effectors, and secondly on robot motion planning for material handling.

In the first category, there are several previous research works in literature that investigate optimising the design of end-effectors for material handling (Mantriota and Messina 2011; Patel et al. 2013; Feng et al. 2013; Tuleja and Sidlovská 2014; Schmalz et al. 2016; Datta et al. 2016). Patel et al. (2013) and Feng et al. (2013) propose actuated end-effectors for material handling, respectively with a linear axis and crossbar mechanism, in order to expedite material handling operations for sheet metal parts. Mantriota and Messina (2011) and Tuleja and Sidlovská (2014) investigate how to calculate the maximum holding force for end-effectors with vacuum-cups. Schmalz et al. (2016) present an approach for automatically dimension grippers based on the properties of the handled objects, the handling environment, the handling device, and the motions for the handling process. Datta et al. (2016) investigate optimising the design of a 7-link gripper with piezoelectric actuation, whilst also optimising the performance of the actuator in the optimisation study. These previous research works are mainly focusing on handling rigid parts, or assume that compliant parts remain rigid during handling. Another issue is that these works typically consider the material handling operations isolated, and not as a part of a manufacturing system. These works are thus not applicable for optimising the design of end-effectors for handling compliant parts, in systems where multiple robots operate in a shared workspace.
Though, Ceglarek et al. (2001) propose an end-effector design methodology for compliant sheet metal parts to minimise the deformations during handling. This is extended later by $\mathrm{Li}$ et al. (2002) with a dexterous part-holding model to more accurately estimate the part deformations. Hoffmann and Kohnhäuser (2002) also propose a generic methodology to calculate the ideal positioning of vacuumcups for the end-effector to hold sheet metal parts, taking into account the dynamic behaviour of the part during handling. Although the part deformations are taken into account, each robotic material handling operation is still considered isolated, instead of being part of a manufacturing system, and thus neglect the environment.

For the second category, material handling motion planning research typically consider a predefined end-effector design, and often also assumes that the handled parts are rigid, even though they are compliant. Pettersson et al. (2007) propose simultaneously optimising the robot motion planning and robot drive-train system parameters. A novel concept for robot installation, drive-train, and motion planning for compact and fast press tending has been proposed by Platzer et al. (2013). Glorieux et al. (2016) propose a trajectory and coordination optimisation methodology for the motion planning of the material handling in multi-stage press lines, to improve the productivity. These previous works use a predefined end-effector design is used and the parts' compliance is not considered. Li and Ceglarek (2002) investigate material handling of compliant sheet metal parts, focusing on time-optimal collision-free trajectory generation whilst avoiding excessive part deformations to maintain the dimensional quality of the parts. However, only a single isolated robot with static obstacles in the workspace is considered.

Table 1 gives an overview of the previous works. A broader survey of model-based manipulation planning of deformable objects is given by Jiménez (2012). It can be concluded that there is currently no available methodology to optimally design end-effectors and motion planning for maximum productivity of material handling with compliant parts in multi-robot systems.

\section{Case study}

In order to illustrate the proposed methodology, it is applied to a specific real-world industrial case study that considers the multi-robot material handling system in a tandem sheet metal press line for multi-stage stamping. The motion planning of these material handling systems is a critical issue for the industry (Glorieux et al. 2015a).

A tandem sheet metal press line includes multiple presses that are placed in a line, and material handling devices (typically robots) that transfer the plates from press to press, 
Table 1 Overview of previous research work

End-effector design

\begin{tabular}{ll}
\hline rigid parts & (Schmalz et al. 2016) \\
& (Tuleja and Sidlovská 2014) \\
& (Feng et al. 2013) \\
& (Patel et al. 2013) \\
& (Ceglarek et al. 2001) \\
compliant parts & (Hoffmann and Kohnhäuser 2002) \\
& (Li et al. 2002) \\
Robot trajectories optimisation & \\
isolated & (Liao and Wang 2003) \\
& (Li and Ceglarek 2002) \\
& (Pettersson et al. 2007) \\
& (Platzer et al. 2013) \\
coordinated & (Glorieux et al. 2016) \\
\hline
\end{tabular}

through the line. The robots' end-effectors use vacuum-cups (or shovels/fingers) to hold sheet metal plates. When a press has performed the stamping operation, one robot unloads the stamped plate and another loads a new unstamped plate into the press as quick as possible.

As shown in Fig. 3, the considered press line uses specialised 2D-belt Binar UniFeeder robots (Binar Olofström AB 2014), or also called H-bot (da Silva 2015), for the material handling. These robots are mounted with two endeffectors to unload the previous press and load the next press in one single motion. These are indicated as end-effector 1 and 2 on robot $r$ in Fig. 3. This makes the multi-robot coordination for each press inter-dependent, which adds to the difficulty for designing the end-effectors and planning the robot motions. Hence, this is a particularly interesting case for this work.

In Fig. 3, robot $r$ simultaneously picks up the plate from Press $r$ - 1 with end-effector 1 and the plate from Table $r$ with end-effector 2 . Next, the plate in end-effector 1 is then placed on Table $r$, whilst at the same time the plate in endeffector 2 is loaded into Press $r$. The advantage of this is that robot $r$ unloads Press $r-1$ and loads Press $r$ in a single motion. More specifically, the operation sequence for a cycle of Press $r$ in Fig. 3 is predefined and is as follows:

1. robot $r$ loads Press $r$ (after unloading Press $r-1$ ),

2. press $r$ performs the stamping operation,

3. robot $r+1$ unloads Press $r$ (and loads Press $r+1$ ),

and this is repeated in the next cycle, for the next plate. It is important to note that an operation can only be started after the robot or press has completed its previous operation(s). This can result in additional time-delays between operations and cycles.

The material handling for the first press in a line is typically most critically and is often a bottleneck for multistage press lines (Ceglarek et al. 2001). The plates that are loaded into that press are usually blank sheet metal plates, i.e. flat rectangular plates. These are relatively more compliant and, in the next stages of the press line, the plates become stiffer and thus less compliant when they are stamped and trimmed. The material handling of blanks is thus particularly suitable for evaluating the proposed methodology. Hence, the considered system in this case study thus includes the first press in the line and the two robots (loading and unloading).

Currently, the industrial practice for this case study is to design the end-effectors in order to minimise the part deformations during handling. The motions for the material handling robots are then planned for these end-effectors by manual tuning, online during production by the operators, at the launch of a new product. The manual tuning is done by ad-hoc trial-and-error. There are several drawbacks with this. Firstly, the end-effectors are designed without taking into account the influence on the multi-robot coordination, which is detrimental for the overall performance of the system. Secondly, ad-hoc trial-and-error is not reliable since it is dependent on the skills and experience of the operator. The resulting productivity can turn out to be significantly lower than expected, which can have costly consequences for the company. Thirdly, online manual tuning requires the press line to be available whilst it is working at a lower
Fig. 3 Illustration of the single press with two robots (loading and unloading) of the considered case study

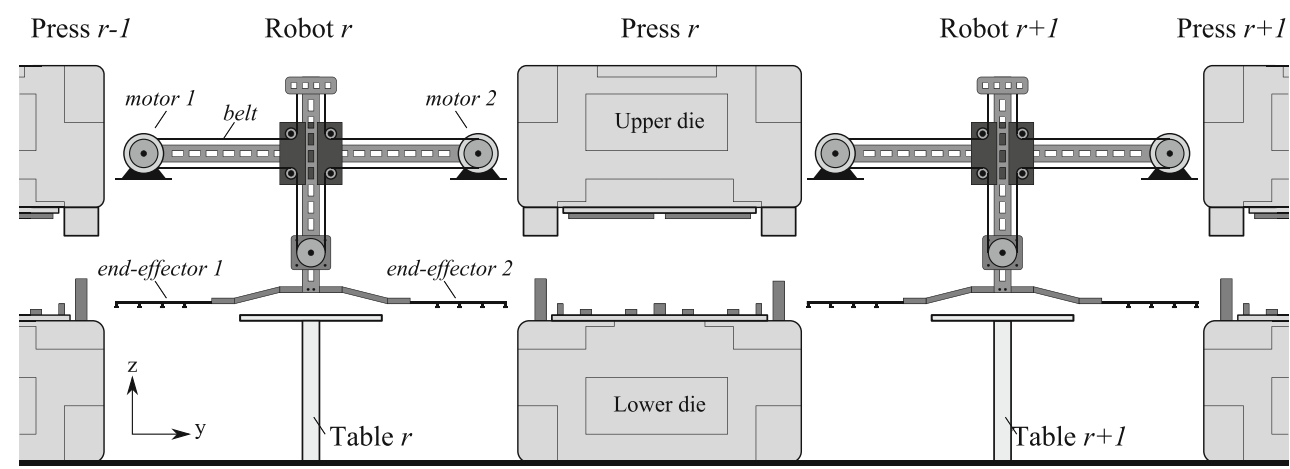


productivity, which is very expensive since these production systems come with a very large tooling and equipment cost.

\section{Problem formulation and modelling}

A general formulation to model the end-effector design and motion planning problem for multi-robot material handling of compliant parts is presented in this section. It is assumed in this work that each operation is assigned to strictly one robot and also that the operation-sequence is predefined, which is typically the case for material handling. This is a multi-disciplinary problem as it includes

1. end-effector's holding force,

2. dynamic part deformations,

3. induced stress,

4. path planning with collision avoidance,

5. trajectory generation,

6. multi-robot coordination.

The challenge is to integrate these different aspects into one problem that can be solved efficiently. As discussed earlier, there are several inter-dependent relationships between the variables which result in implicit constraints for the problem. The novelty of the presented problem formulation compared to the work by Schmalz et al. (2016) is that the end-effector design and the motion planning for the material handling operations are co-adapted, whereas Schmalz et al. (2016) focused on the end-effector design and considered the motions as a given.

\subsection{Objective function}

It is proposed to consider two objectives during the optimisation, but prioritising the one over the other. The primary objective is to minimise the cycle-time in order to improve the productivity, and the secondary is to minimise the part deformations during handling to avoid the risk of affecting the dimensional quality. The objective function is formulated as follows

$\min \boldsymbol{F}_{o}\left(\mathbf{Q}^{r}, \mathbf{A}^{r}, \mathbf{V}^{r}, H^{r} \mid \forall r=1, \ldots, R\right)$

where $\boldsymbol{F}_{o}=\left\{F_{1}, F_{2}\right\}$ is the set of prioritised objectives with $F_{1}$ and $F_{2}, \mathbf{Q}^{r}=\left\{\mathbf{q}\left(1^{r}\right), \ldots, \mathbf{q}\left(s_{n}^{r}\right)\right\}$ is a set with $s_{n}^{r}$ vectors specifying the joints' position, and thereby the location and orientation of the end-effector, for each segment $s^{r}$ for robot $r, s_{n}^{r}$ is the number of segments for the complete path, $\mathbf{V}^{r}$ and $\mathbf{A}^{r}$ are respectively the velocities and accelerations, $H^{r}$ is the end-effector design, $R$ is the number of robots. Objective $F_{1}$ gives the cycle-time and has a higher priority than objective $F_{2}$ that gives the part deformations. These two objectives are formulated as follows

$$
\begin{aligned}
& C T=F_{1}\left(\mathbf{Q}^{r}, \mathbf{A}^{r}, \mathbf{V}^{r}, H^{r} \mid \forall r=1, \ldots, R\right) \\
& u=F_{2}\left(\mathbf{Q}^{r}, \mathbf{A}^{r}, \mathbf{V}^{r}, H^{r} \mid \forall r=1, \ldots, R\right)
\end{aligned}
$$

where $C T$ is the cycle-time of the considered system, and $u$ are the part deformations during handling. The secondary objective $\left(F_{2}\right)$ that considers the part deformations $(u)$ becomes relevant when comparing solutions with an equal cycle-time $(C T)$ for the primary objective $\left(F_{1}\right)$. This is to handle scenarios where there are many solutions that give the same optimal cycle-time. This typically occurs when a specific operation in the system forms a bottleneck, i.e. all other operations have to wait until it is completed before the next cycle can be started. The secondary objective $\left(F_{2}\right)$ then guides the optimisation to the time-optimal solution that also minimises the part deformations during handling.

\subsection{Optimisation variables}

The optimisation variables are the robot path $\mathbf{Q}^{r}$, velocities $\mathbf{V}^{r}$ and accelerations $\mathbf{A}^{r}$, and the end-effector designs $H^{r}$ for each robot in the systems. The values for these variables must be within certain ranges or equal to specific values in order to agree with the considered problem. This is expressed in the model by boundary conditions. The first boundary conditions specify that the robot paths must go from the pick to the place location and back. This can be formulated as follows

$$
\begin{aligned}
& \mathbf{q}\left(s_{\text {pick }}^{r}\right)=\mathbf{q}_{\text {pick }}^{r} \\
& \mathbf{q}\left(s_{\text {place }}^{r}\right)=\mathbf{q}_{\text {place }}^{r}
\end{aligned}
$$

where $\mathbf{q}_{\text {pick }}^{r}, \mathbf{q}_{\text {place }}^{r}$ are respectively the pick and place locations for robot $r$, and $s_{\text {pick }}^{r}, s_{\text {place }}^{r}$ are respectively the pick and place segment of the trajectory. The robot velocity and acceleration when picking or placing a part must be zero in order for the end-effector to securely grip or release the part. This is expressed by the following boundary conditions

$$
\begin{array}{ll}
\mathbf{v}\left(s_{\text {pick }}^{r}\right)=\phi ; & \mathbf{v}\left(s_{\text {place }}^{r}\right)=\phi ; \\
\mathbf{a}\left(s_{\text {pick }}^{r}\right)=\phi ; & \mathbf{a}\left(s_{\text {place }}^{r}\right)=\phi ;
\end{array}
$$

where $\mathbf{v}\left(s^{r}\right)$ and $\mathbf{a}\left(s^{r}\right)$ are respectively the velocity and acceleration vectors of robot $r$ during segment $s^{r}$. Similar boundary conditions are required to guarantee that the robot stands still at the start and the end of its trajectory, which are formulated as follows

$$
\begin{array}{ll}
\mathbf{v}\left(1^{r}\right)=\phi ; & \mathbf{v}\left(s_{n}^{r}\right)=\phi ; \\
\mathbf{a}\left(1^{r}\right)=\phi ; & \mathbf{a}\left(s_{n}^{r}\right)=\phi ;
\end{array}
$$

to ensure that the robot's velocities and accelerations are zero. The next boundary conditions ensure that the velocity, 
acceleration, and torque of the robot are within the allowed range of the robot along the entire trajectory

$$
\begin{aligned}
& \mathbf{v}_{\text {min }}^{r}<\mathbf{v}\left(s^{r}\right)<\mathbf{v}_{\text {max }}^{r} \\
& \mathbf{a}_{\text {min }}^{r}<\mathbf{a}\left(s^{r}\right)<\mathbf{a}_{\text {max }}^{r} \\
& \tau_{\text {min }}^{r}\left(\mathbf{v}\left(s^{r}\right)\right)<\tau\left(s^{r}\right)<\tau_{\text {max }}^{r}\left(\mathbf{v}\left(s^{r}\right)\right)
\end{aligned}
$$

where $\mathbf{v}_{\text {min }}^{r}, \mathbf{v}_{\text {max }}^{r}, \mathbf{a}_{\text {min }}^{r}$ and $\mathbf{a}_{\text {max }}^{r}$ are respectively the minimum and maximum velocity and acceleration vectors for the joints of robot $r, \tau\left(s^{r}\right)$ is the torque load vector at segment $s^{r}$, and $\tau_{\text {min }}^{r}\left(\mathbf{v}\left(s^{r}\right)\right)$ and $\tau_{\text {max }}^{r}\left(\mathbf{v}\left(s^{r}\right)\right)$ are respectively the velocity dependent minimum and maximum torque vectors for the joints with motors and gears of robot $r$. It must be noted that rather often $\mathbf{v}_{\text {min }}^{r}=-\mathbf{v}_{\text {max }}^{r}$, and similarly for the acceleration and torque limits. These boundaries need to be integrated as constraints in the model in order to prevent planning motions that require a larger torque than that the joints of the robots are able to deliver (Pettersson et al. 2007).

Finally, $H^{r}$ is a set that includes all the optimisation variables for the design of the end-effectors. For the example of end-effectors with vacuum-cups, the variables in $H^{r}$ could determine the coordinates for the location of the vacuum-cups relative to the handled part(s).

\subsection{Design constraints}

\subsubsection{Forces}

In order to calculate the part deformations, and the holding force of the end-effector it is necessary to model the force on the part during handling. The force on the part is influenced by the robot's motion and is formulated as follows

$\mathbf{F}^{r}=F_{3}\left(\mathbf{Q}^{r}, \mathbf{V}^{r}, \mathbf{A}^{r}\right)$

where $\mathbf{F}^{r}$ is a vector with the force on the part during each segment $s$, and the function $F_{3}$ represents its relationship with the robot $r$ 's path $\mathbf{Q}^{r}$ (gravitation force), velocities $\mathbf{V}^{r}$ (air resistance force) and accelerations $\mathbf{A}^{r}$ (inertia force). In the case that the orientation of the end-effector does not change along the path, the direction of the gravitation force is constant and can be integrated with the inertia force. This is the case in the press line example, where the handled sheet metal plates are always horizontal during handling. The total force $\mathbf{F}^{r}$ on the part can then be formulated in more detail as follows

$\mathbf{F}^{r}=\mathbf{F}_{\text {inertia }}^{r}+\mathbf{F}_{\text {air }}^{r}$

where $\mathbf{F}_{\text {inertia }}^{r}$ is calculated as follows

$\mathbf{F}_{\text {inertia }}^{r}=m_{p}^{r} \cdot\left(g+\mathbf{A}^{r}\right)$ where $g$ is the gravitational acceleration, and $m_{p}^{r}$ is the mass of the part handled by robot $r$. The air resistance force $\mathbf{F}_{a i r}^{r}$ is calculated as follows

$\mathbf{F}_{a i r}^{r}=\frac{1}{2} \cdot \rho_{a} \cdot\left(\mathbf{V}^{r}\right)^{2} \cdot C_{d}^{r} \cdot A_{x y}^{r}$

where $\rho_{a}$ is the density of air, $C_{d}^{r}$ is the drag coefficient for the (undeformed) geometry of the part, and $A_{x y}^{r}$ is the area in xy-plane of the (undeformed) part handled by robot $r$. For a flat thin plate perpendicular to air flow, and the drag coefficient $C_{d}$ is equal to 2 .

\subsubsection{Deformations and stresses}

Based on the calculated force on the part, the deformation of the part during handling can be approximated whilst also taking into account the end-effector' design. The dynamic part deformations along the trajectory can typically be calculated by performing a transient response analysis of the vibrations using FEA (Zienkiewicz and Taylor 2000). The following relationship must then be formulated in the model

$\mathbf{u}_{g}^{r}=F_{4}\left(\mathbf{F}^{r}, H^{r}\right)$

where $\mathbf{u}_{g}^{r}$ is a vector with the dynamic deformations of the part, and function $F_{4}$ represents the relationship between the force on the part and the end-effector design, and the part deformations.

The induced stress in the part due to the deformations is an important aspect since it is the stress that indicates whether a deformation is elastic or plastic (permanent). Hence, only the maximum stress in the deformed part is of interest. The formulation of the model needs to include the following relation

$\sigma_{\text {max }}^{r}=F_{5}\left(\mathbf{u}_{g}^{r}\right)$

where $\sigma_{\text {max }}^{r}$ is a vector with the maximum stress along the trajectory, and function $F_{5}$ represents the relationship with the dynamic deformation of the part, and the maximum induced stress.

\subsubsection{Holding force constraint}

An end-effector typically has a maximum holding force. If the required force to hold the part exceeds this maximum holding force during handling, the end-effector will end up dropping the part. Hence, the next constraint is necessary to prevent this, which is formulated as follows

$\mathbf{F}_{h}^{r} \leq F_{h, \text { max }}^{r}\left(H^{r}\right)$

where $\mathbf{F}_{h}^{r}$ is the required force to hold the part, and $F_{h, \text { max }}^{r}\left(H^{r}\right)$ is the maximum holding force for end-effector design $H^{r}$ of robot $r$. The force to hold the part will be equal to the force on the part, i.e. $\mathbf{F}_{h}^{r}=\mathbf{F}^{r}$, for most scenarios. Hence, (8) is used to calculate $\mathbf{F}_{h}^{r}$. 
When the end-effector layout is asymmetric to the part's centre of gravity, there is an additional momentum force, which reduces its maximum holding force. The maximum holding force of the end-effector is therefore thus dependent on its design $\left(H^{r}\right)$, i.e. $F_{h, \max }^{r}\left(H^{r}\right)$. Tuleja and Sidlovská (2014) and Mantriota and Messina (2011) propose how to calculate the maximum holding force of respectively tangential loads and symmetric/asymmetric layouts around the part's centre of gravity for end-effector designs with vacuum-cups. The maximum holding force of the endeffector in the model is calculated based on these works.

\subsubsection{Yield stress constraint}

It is necessary to avoid permanent plastic deformations of the part during handling, as these would damage the part. As mentioned earlier, the stress in the part should not exceed the yield stress of the part's material to avoid plastic deformations. The model formulation therefore needs to include the following constraint

$\sigma_{\text {max }}^{r} \leq \sigma_{y}^{r}$

where $\sigma_{y}^{r}$ is the yield stress of the part's material handled by robot $r$.

Finite Element Analysis (FEA) is used in this work to represent the relationship between the force on the part, the dynamic deformations and the induced stress. The FEA estimates the part deformations and the induced stress. As discussed during the formulation of (14), only the maximum stress is relevant.

FEA is typically rather computationally expensive, particularly for the purpose of optimisation where the analysis needs to be re-run for each evaluation. To avoid this issue, a Response Surface Model (RSM) is constructed based on precalculated samples of the FEA. This RSM is then used to represent the relationship between the force on the part and the maximum stress during the optimisation, within an acceptable accuracy. The formulation in the model is then as follows

$\sigma_{\max }^{r}=f_{R S M}\left(\mathbf{F}^{r}, H^{r}\right)$

where $f_{R S M}$ is a function that represents the RSM, which combines (11) and (12).

To construct the RSM, a set of data samples is generated where each sample has different values for the input parameters, i.e. end-effector designs and the force on the part. Depending on the application range for these parameters, the samples are obtained by for example random (done in this work) or uniform sampling, or by using advanced space mapping methods (Wang et al. 2017; Jansson et al. 2003; Shan and Wang 2010). A physics-driven model (with a FEA-based kernel) is solved for each sample to calculate the corresponding output value for the maximum induced stress (Franciosa et al. 2014).
The RSM is constructed by performing a regression analysis of the samples. The selection of the regression technique varies depending on the complexity and non-linearity of the problem. Non-linear polynomial fitting is used in this work, as proposed by Li and Ceglarek (2002), and the accuracy is verified by non-exhaustive leave- $p$-out cross validation.

\subsubsection{Collision constraint}

The path planning needs to ensure that there will be no collisions between the robot (i.e. robot structure, end-effector, handled part) and the obstacles in the workspace. This constraint formulates this in the model

$g_{1}=g_{1}\left(\mathbf{Q}^{r}, \mathbf{u}_{g}^{r}, H^{r}\right)$

where $g_{1}$ stands for that the path is collision-free concerning (static) obstacles in the workspace. The dynamic deformations of the part during handling are considered for the collision detection to accurately anticipate for all possible interactions between the deformed shape of the part and the obstacles. Mapping the possible collisions into zones in the robot's workspace, in advance, avoids the need for computationally expensive 3D-collision detection simulations.

\subsubsection{Time-delay coordination constraint}

The time-delay coordination constraint is concerned with the final multi-robot operation coordination in the shared workspace to avoid collisions between the robots. This constraint can only be verified when all robot paths and trajectories are defined. In this work, a specific time-delay to start an operation is used to avoid collisions with other robot(s). This is expressed as follows

$g_{2}=g_{2}\left(\mathbf{Q}^{r}, \mathbf{A}^{r}, \mathbf{V}^{r}, H^{r} \mid \forall r=1, \ldots, R\right)$

where $g_{2}$ stands for the collision-free condition concerning all robot operations and that results in the shortest cycletime.

\subsection{Model assumptions}

A few assumptions are made for modelling the end-effector design and multi-robot motion planning problem in this paper. It is assumed that the end-effector and robots' structures can be considered as rigid because the deformations are negligible in comparison with those of the handled part. Furthermore, it is also assumed that the handled parts are all identical, and these are always located at the exact same location from where it is initially picked up by the first robot in the sequence of operations. After the maximum stress is verified to be less than the yield stress of the material, the 
elastic deformation are calculated under the assumption that these are linear. It is also assumed that phenomena such as aeroelastic flutter do not occur when handling compliant parts such as sheet metal plates.

\section{Proposed methodology}

The proposed methodology is developed to solve the problem formulated in Section 4, and thus to find the optimal design for the structure of the end-effectors and to co-adapt the motion planning for the multi-robot material handling systems with compliant parts. The proposed optimisation methodology makes use of an Asymmetric Subspace Optimisation (ASO) architecture according to the classification proposed in the survey of multi-disciplinary design optimisation architectures presented by Martins and Lambe (2013). The choice for ASO is motivated by that the collision detection simulation for the multi-robot coordination requires an order of magnitude more time to complete than evaluating the other constraints concerning the robot trajectories. Henceforth, it becomes beneficial to tune the trajectories within an inner optimisation loop by utilising the ASO architecture (Martins and Lambe 2013). The eXtended Design Structure Matrix (XDSM) diagram (Lambe and Martins 2012) of the ASO architecture for the proposed methodology is shown in Fig. 4. The XDSM diagram shows both the data dependencies (thick grey lines) and process flows (black thin dashed lines indicate the inner loop, and black thin full lines the outer loop). In the diagram, the rectangles represent the process steps of the methodology, the parallelograms show data inputs and outputs, and the rounded rectangles indicate drivers that control the iterative processes.

In the model formulation, the optimisation variables are the parameters of the motion planning $\left(\mathbf{Q}^{r}, \mathbf{V}^{r}, \mathbf{A}^{r}\right)$ and the design of the end-effectors $\left(H^{r}\right)$. A solution refers to a set of values for the optimisation variables. During the optimisation, different trial-solutions are iteratively generated by an optimisation algorithm. The formulated model is then used to determine the objective function values of each solution, using its values. As defined in the formulation of the objective function in (1), the cycle-time is the primary objective function value, and the maximum part deformation is the secondary. Only feasible solutions, which are solutions that agree with all constraints of the problem, are considered during the optimisation and infeasible ones are directly discarded. When comparing the objective function values of the different feasible solutions, the cycle-time is prioritised, meaning that a solution with a shorter cycle-time is always considered as better than others with a longer cycle-time. Only when the cycle-time of the solutions is equal, the one with a smaller maximum deformation is considered better.
In other words, a solution $S_{1}$ is considered better than a solution $S_{2}$ if one of the following two statements holds true

$$
\begin{aligned}
& C T\left(S_{1}\right)<C T\left(S_{2}\right) \text { or } \\
& C T\left(S_{1}\right)=C T\left(S_{2}\right) \text { and } u_{\max }\left(S_{1}\right) \leq u_{\max }\left(S_{2}\right)
\end{aligned}
$$

where $C T\left(S_{i}\right)$ represents the cycle-time with solution $S_{i}$ and $u_{\max }\left(S_{i}\right)$ the maximum deformation of the handled parts with solution $S_{i}$.

The proposed optimisation methodology consists of two nested loops, an outer- and an inner-loop. The outer-loop includes the optimisation algorithm and handles generating the end-effector designs and robot paths, and the time-delay coordination after the trajectories are determined during the inner-loop. The outer-loop is thus concerned with only the optimisation variables related to the end-effector designs and robot paths, i.e. $\mathbf{Q}^{r}$ and $H^{r}$. The inner-loop handles generating, tuning and verifying the trajectories and is thus concerned with only the variables related to the robots' velocities and accelerations, i.e. $\mathbf{V}^{r}$ and $\mathbf{A}^{r}$.

\subsection{Outer-loop}

The outer-loop starts with the optimisation algorithm, which generates different solutions for the variables $\mathbf{Q}^{r}$ and $H^{r}$. One iteration of the outer-loop relates to the evaluation of a solution. It starts with generating the end-effector designs and the robot paths according to the values of the trialsolution that is under evaluation. More specifically, for the end-effector designs, this includes representing the geometries for the collision detection simulation, and how these hold the handled plates for the FEA. This furthermore includes generating and verifying the paths.

Next, the inner-loop is deployed to determine the velocities and accelerations for the trajectory of each robot along its path. The result of the inner-loop are tuned trajectories that are verified for the holding force, collisions, and plastic deformations. The inner-loop calculates the objective function value for maximum deformation $\left(u_{\max }\right)$. Thereafter, when verified trajectories for all operations are available, the outer-loop continues.

The next step is to evaluate the time-delay coordination constraint (17) to determine the timings for starting the different operations according to the predefined sequence so that the robots do not collide with each other. Using fast collision detection simulation with 3D CAD models of the geometries of the robots following the planned trajectories, the possible collisions between the robots along their trajectories are determined and represented in a position-based relative collision mapping, i.e. in the coordination space (Kavraki and LaValle 2008). The coordination method proposed by Bien and Lee (1992) is then used to determine the minimal time-delay to avoid all the possible collisions. Using the relative collision mapping between robots in the 


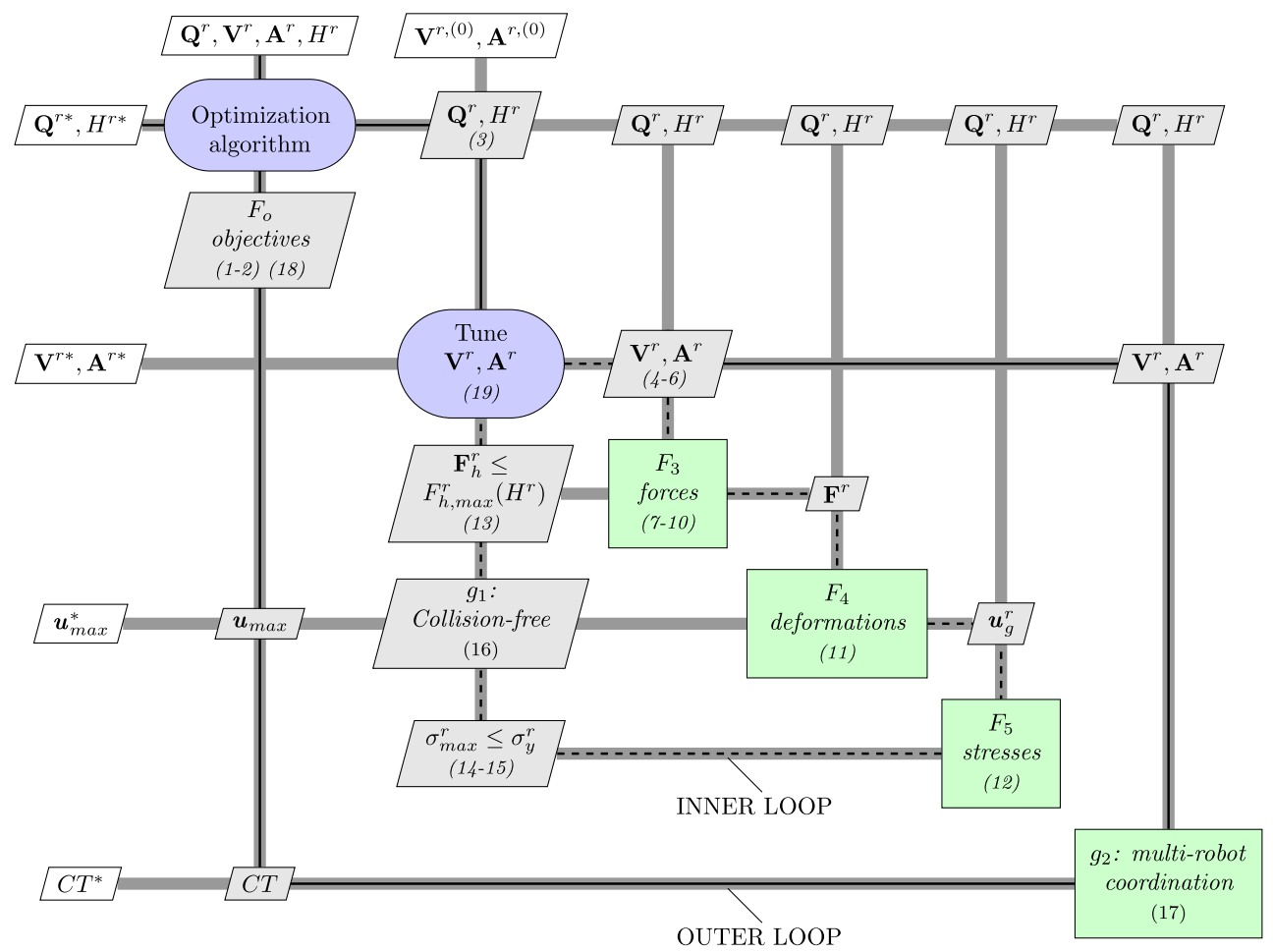

Fig. 4 XDSM Diagram for the ASO architecture of the proposed optimisation methodology (dashed line indicate the inner loop, and full line the outer loop of the proposed methodology)

coordination space, the coordination method effectively calculates the minimal time-delay between the start of the two robots so that these will not collide. This is done for each pair of robots that perform consecutive operations in the shared workspace. These minimal time-delays are afterwards adjusted to take into account the availability of the robots, i.e. that a robot can only start an operation when it has completed its previous operation. Combining the resulting time-delays for all operations gives the system's cycle-time. The cycle-time, together with the maximum deformation value $\left(U_{\max }\right)$, are returned to the optimisation algorithm.

\subsection{Inner-loop}

The task of the inner-loop is thus to generate, tune and verify the trajectory of each robot operation along the provided path that was generated earlier in the outer-loop. Hence, it is concerned with determining the values that specify the optimisation variables $\mathbf{V}^{r}$ and $\mathbf{A}^{r}$. Since, the primary objective is reducing the cycle-time, the trajectories are initially generated at the maximum velocity and acceleration of the robot.

Based on the simulated trajectory, the force on the part, the maximum stress, and the part deformations are calculated respectively using (7), (15), and (11). This provides the information to verify the holding force constraint (13), the yield stress constraint (14), and the collision constraint (16). As presented in the problem formulation, these are proportional with the velocity and/or acceleration of the robot along the trajectory. Therefore, when one of these constraints is violated, the velocity and acceleration for the trajectory are adaptively tuned in order to remove the violation. Reducing the velocity and acceleration will reduce the force on the part, the maximum induced stress, the part deformations. The velocity and/or acceleration is decreased for the corresponding segment of the trajectory where the constraint violation occurs. The tuning of the velocity and acceleration are done as follows

$$
\begin{aligned}
& \mathbf{v}_{\text {new }}\left(\left[s_{s}^{r} \ldots s_{e}^{r}\right]\right)=\mathbf{v}_{\text {old }}\left(\left[s_{s}^{r} \ldots s_{e}^{r}\right]\right)-\varepsilon_{v} \\
& \mathbf{a}_{\text {new }}\left(\left[s_{s}^{r} \ldots s_{e}^{r}\right]\right)=\mathbf{a}_{\text {old }}\left(\left[s_{s}^{r} \ldots s_{e}^{r}\right]\right)-\varepsilon_{a}
\end{aligned}
$$

where $\left[s_{s}^{r} \ldots s_{e}^{r}\right]$ are the indices of the trajectory segments where the constraint violation occurs, and $\varepsilon_{v}$ and $\varepsilon_{a}$ are the step-sizes for decreasing respectively the velocity and accelerations for the tuning.

The trajectory is then re-generated for the tuned velocities and accelerations, and the constraints are then reverified. The tuning of the velocities and accelerations is repeated until the trajectory agrees with all constraints, or until the minimum velocity or acceleration is reached. In the latter case, the solution is declared infeasible since it is impossible to generate trajectories that agree with all constraints. 
When successful, the inner-loop generates a verified trajectory along the provided path for each robot operation, at the highest possible velocity and acceleration according to the constraints. The velocity and acceleration is as high as possible to reduce the duration of the operation, because reducing the cycle-time is the primary objective. The proposed optimisation methodology then continues with the outer-loop.

\section{Implementation}

This section presents the implementation of the proposed methodology for the case study. The sheet metal part in the case study is an inner side panel of the floor frame for a car body. The plate's material is DP800 steel. Some optimisation variables can only be parametrised indirectly for the case study because these are not directly accessible, which is common with real-world systems. A robot path $\left(\mathbf{Q}^{r}\right)$ is specified by "via-locations" ( $y z$-coordinates and zone radius), indicated as $\mathrm{L}_{1 \ldots 4}$ in Fig. 5. Based on these via-locations, the path is then generated according to the robot controller, using a model. For the robots in the press line, there are four via-locations that, together with the pickand place-locations, make up the path.

Furthermore, the parametrisation of the end-effectors $\left(H^{r}\right)$, which defines the locations of the vacuum-cups on the plate is illustrated in Fig. 6. The end-effectors always have two vacuum-cups in the case study, one at coordinates $\left(x_{1}, y_{1}\right)$ and another one at $\left(x_{2}, y_{2}\right)$. For constructing the RSM of the Yield Stress Constraint, it was found that 100 samples and using a $4^{\text {th }}$ degree polynomial provides sufficient accuracy, i.e. the root-mean-squared error (RMSE) is $16.35 \mathrm{MPa}$ and the normalised RMSE is 0.061 .

\subsection{Outer loop}

The outer loop starts with the optimisation algorithm, which is the Constructive Cooperative Coevolutionary Differential Evolution $\left(\mathrm{C}^{3 i} \mathrm{j}\right.$ DErpo) proposed by Glorieux et al. (2015b).

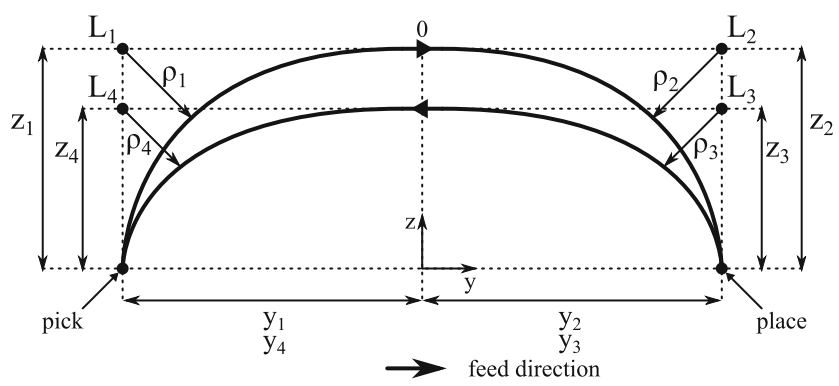

Fig. 5 Illustration of the robot path for the material handling in the press line

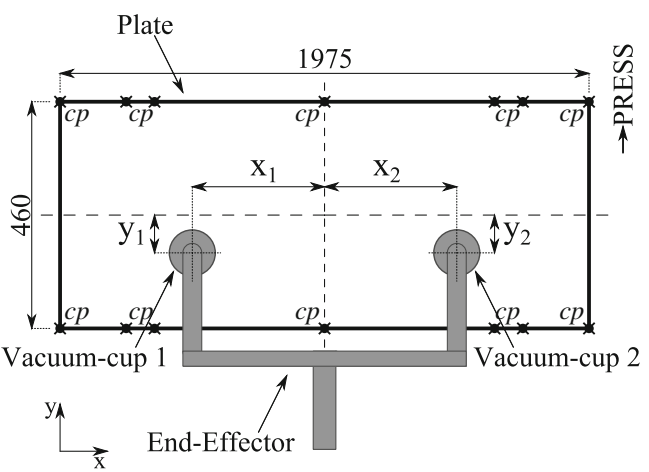

Fig. 6 Top-view of the handled blank sheet metal plate and the end-effector design ( $c p$ indicates the critical points for the collision detection)

The problem needs to be decomposed into subproblems in advance for the $\mathrm{C}^{3 i} \mathrm{j}$ DErpo algorithm. For the case study, each subproblem corresponds to a robot in system. $\mathrm{C}^{3 i}$ jDErpo optimises the subproblems separately in cooperative fashion in order to co-adaptively attune the optimisation variables of the subproblems and thereby consider the inter-dependencies between them. $\mathrm{C}^{3 i} \mathrm{j}$ DErpo is particularly suitable for large-scale optimisation problems with inter-dependent subproblems, i.e. non-fully separable subproblems (Glorieux et al. 2015b). This makes it suitable for the press line case study, as show by Glorieux et al. (2015a). The population size is 20 , and the termination criterion is 1000 evaluations.

The evaluation of a solution starts with generating the robot paths $\left(\mathbf{Q}^{r}\right)$, based on the specified parameters (eight per robot) for the via-locations. This is done accurately using a model of the 2D-belt Binar Unifeeder robot controller. A description of this model is given by Glorieux et al. (2015c). The end-effector designs are generated based on the parameters (two per end-effector) that specify the designs' variables $\left(H^{r}\right)$.

In the final step of the outer loop, the time-delays are calculated for the multi-robot coordination. The collision detection simulation is done using the Proximity Query Package (PQP) (Larsen et al. 2000), which is a software library for proximity queries, including collision detection of a pair of geometric models composed of triangles. The used geometric models are the 3D CAD models of the geometries of the robots and press dies in the real-world press line. These are simplified to only consider the relevant geometrical elements in order to expedite the collision detection simulation.

\subsection{Inner loop}

The inner loop starts with generating the trajectories at maximum velocity $\left(\mathbf{V}^{r}\right)$ /acceleration $\left(\mathbf{A}^{r}\right)$ according to the constraints (4)-(6) and a model of the robot (Glorieux et al. 
2015a). It then calculates the force on the part during handling. Since they are flat blank sheet metal plates that are always held horizontally, it can be assumed that the force in vertical direction has a significantly larger influence than the force in horizontal direction. The presented formulation focuses therefore only on the vertical force, which is the combination of the acceleration-caused inertia force (i.e. gravity and robot accelerations) as in (9), and the air resistance force as in (10). The diameter of the end-effectors' vacuum-cups is $105 \mathrm{~mm}$ and the maximum holding force is $236 \mathrm{~N}$ in normal direction (i.e. $z$-axis). Both the holding force and yield stress constraint can then be verified when the force on the part is known.

Next is the transient response analysis to estimate the dynamic deformation of the part during handling. This also gives the maximum part deformation objective function value. The Variation Response Method (VRM) (Franciosa and Ceglarek 2016), a physics-driven modeller with FEAbased kernel, is used in this work because of its advanced capabilities to parametrise key aspects including the loading force, part's compliance, end-effector, and vacuum-cups (Franciosa and Ceglarek 2015; Franciosa et al. 2016). The vacuum-cups are modelled as a set of rigid points in a circular pattern. It is assumed that the positions of the vacuum-cups relative to the plate are fixed during handling and are identical for each cycle. To reduce the FEA's computation time, only the deformations of critical points on the plate are calculated. These critical points are the first to interfere with the obstacles or other robots when colliding. The critical points (shown in Fig. 6) are on the plate's outer border at the corners, at the centres in $x$-direction, and where it will collide with the guiding pins of the lower die (as shown in Fig. 1).

The next step is the collision detection simulation between the obstacle(s) and the dynamic deformation of the part, end-effector and robot, which is also done using the PQP library (Larsen et al. 2000).

If the constraints are not met, the next step is tuning the corresponding robot's velocity and acceleration along the trajectory. The velocity and acceleration in the corresponding segment (five per operation) of the trajectory are tuned according to (19). When all constraints are met, the trajectories are returned to the outer loop for the multi-robot coordination.

\section{Tests}

Several tests have been performed to illustrate the purpose and the advantage of the proposed methodology. More specifically, the goal is to demonstrate the gain in productivity by co-adaptively designing the end-effectors with the robot motion planning, i.e. the contribution of the proposed methodology. Secondarily, the tests also demonstrate that it is critical to consider the deformations of the compliant parts in order to plan reliable robot motions for the multirobot system in this case study, i.e. the need for the proposed methodology. Three different tests are performed for this. The optimisation for each test has been repeated 10 times in order to obtain a statistically reliable result, which is necessary because the used optimisation algorithm is stochastic. In the first test, the methodology is deployed as proposed for the considered case study.

In the second test, the robot motions are planned for compliant parts but using a default end-effector design, which is based on the end-effector that is used in the real-world press line, with $x_{1}, x_{2}=493.75 \mathrm{~mm}$ and $y_{1}, y_{2}=0.0 \mathrm{~mm}$. Only the via-locations of the path and the robot trajectory variables (velocities and accelerations) are optimised in this test. This provides a reference for a comparison with the first test to demonstrate the importance of the optimised end-effector designs.

In the third test, the robot motion planning is performed assuming that the parts are rigid and using the default endeffector design. The yield stress constraint from (14) is thus ignored in this test. The found optimal solution in this test is analysed to reveal the consequences of assuming that the parts are rigid, which demonstrates the need for considering the deformations of the compliant parts during handling.

\section{Results and discussion}

This section discusses and compares the results of the three different tests performed in this work. The results of the optimal solution found by the optimisation are the objective function values for the maximum part deformation $u_{\max }$ and the cycle-time $C T$. The mean and standard deviation (std) of the results across the repetitions are shown in Table 2, where the productivity of the solutions is also given as production rate $(P R)$, which is expressed in parts per minute ([pr/min $]$ ).

The optimal solution in Test 1 has an end-effector design with $x_{1}, x_{2}$ equal to $516.6 \mathrm{~mm}$ and $y_{1}, y_{2}$ equal to 87 $\mathrm{mm}$. The location of the vacuum-cups on the plate are thus

Table 2 Comparison of the part deformation $\left(u_{\max }\right)$, cycle-time $(C T)$, and production rate $(P R)$ results, (result of Test 3 is infeasible)

\begin{tabular}{|c|c|c|c|c|c|c|}
\hline \multirow[t]{2}{*}{ Test } & \multicolumn{2}{|c|}{$u_{\max }[\mathrm{mm}]$} & \multicolumn{2}{|l|}{$\mathrm{CT}[\mathrm{s}]$} & \multirow{2}{*}{$\begin{array}{l}\mathrm{PR} \\
{[\mathrm{pr} / \mathrm{min}]}\end{array}$} & \multirow{2}{*}{$\begin{array}{l}\Delta \mathrm{PR} \\
{[\%]}\end{array}$} \\
\hline & mean & std & mean & std & & \\
\hline 1 & -25.2 & 7.6 & 4.074 & 0.005 & 14.73 & 0.0 \\
\hline 2 & -21.8 & 0.5 & 4.153 & 0.003 & 14.45 & -1.9 \\
\hline 3 & -22.1 & 0.5 & 4.132 & 0.003 & 14.52 & -1.4 \\
\hline $3 a$ & -21.2 & 0.4 & 4.252 & 0.012 & 14.11 & -4.2 \\
\hline
\end{tabular}


moved to the outside of the plate in $x$-direction and away from the centre of the plate in $y$-direction. When comparing Test 1 with Test 2, it can be seen in Table 2 that there is a relatively small difference between the maximum part deformation. This was further investigated by FEA and visualised as shown in Fig. 7, together with the difference in induced stress. Table 2 also shows that the cycle-time is longer in Test 2 than in Test 1, which results in a productivity that is $1.9 \%$ higher in Test 1 . Although this difference in productivity might seem minor at first sight, this is nevertheless a substantial improvement considering that the press line is used for high-volume production. In fact, the $1.9 \%$ increase in productivity means that 134 more parts are produced during 8 hours. This thus shows that the productivity is significantly improved by designing the end-effector co-adaptively with the multi-robot motion planning.

When looking at the optimal solution found in Test 3 in Table 2, it is very interesting to see that its productivity is lower than Test 1 (i.e. $-1.4 \%$ ), and only slightly higher than Test 2 . Note that the only difference between Test 2 and 3 is the parts are assumed to be rigid. This indicates that, in Test 1 , the increased productivity with the optimised end-effector designs is mainly related to the shorter time-delays for the multi-robot coordination, and less to the part deformations or holding force. This further confirms the contribution of the proposed methodology, i.e. designing the end-effectors co-adaptively with multi-robot motion planning.

The optimal solution found in Test 3 is also further analysed by simulating it without the assumption that the parts are rigid. This showed that there are multiple collisions between the deformed part and the lower-die obstacle. Additionally, the maximum induced stress in the part due to the dynamic deformations exceeds the yield stress of the material. Hence, the handled part would undergo plastic deformations that damage it. This shows that planning the motions whilst considering the parts as rigid is highly unreliable.

An additional analysis of the optimal solution in Test 3 is performed to replicate the effect of Test 3 for an industrial scenario. Modifying the robot paths is an elaborate task, whereas changing the robot velocity can typically easily be done online. Hence, for this scenario, only the robot velocities are tuned to avoid those problems (i.e. collisions and plastic deformations) in Test 3, and then the multi-robot coordination is consequently adjusted. The result of this is shown as Test $3 \mathrm{a}$ in Table 2. It can be seen that the productivity is now significantly lower, i.e. $-4.2 \%$ compared to Test 1 . Unreliable motion planning can result in a substantially lower productivity than anticipated for during the planning, which is a problematic issue for the industry.

The proposed methodology addresses the lack of reliable systematic methods to design the end-effectors whilst coadaptively plan the material handling motions in order to improve the productivity and maintain the products' dimensional quality. There are several advantages compared to the current industrial practise. Besides the improved productivity, the proposed methodology relies on digital tools (i.e. models, simulations, and algorithms) and can thus be used offline before launching the production of a new part. It avoids the need for the time-consuming ad-hoc online trialand-error, which is very expensive for press lines such as in the case study. In other words, the proposed methodology provides right-first-time solutions for the motion planning and end-effectors design.

Although the case study only considers a single isolated press with two robots, the proposed methodology is directly applicable to entire press lines with multiple presses and press tending robots, with additional coordination between the unloading and the loading of consecutive presses.
Fig. 7 Comparison maximum part deformation with a default and $\mathbf{b}$ optimised end-effector design, and comparison of the induced stress with $\mathbf{c}$ default and d optimised end-effector design (red dots indicate the vacuum-cups)

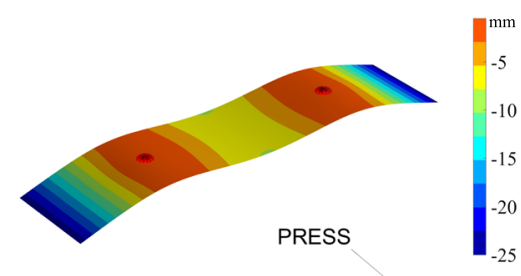

(a)

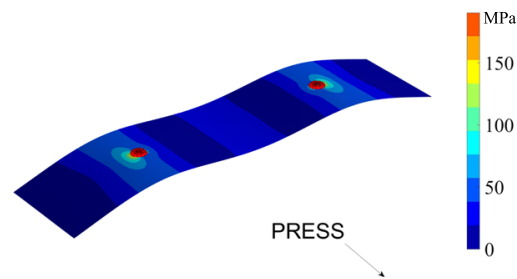

(c)

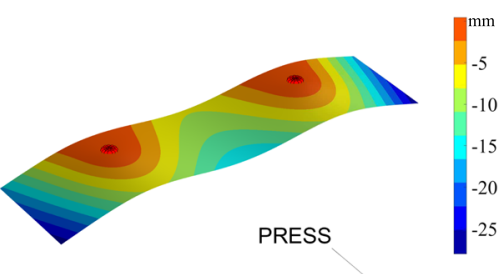

(b)

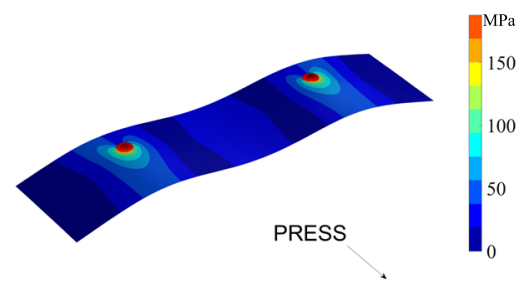

(d) 


\section{Summary and conclusions}

Multi-robot material handling of compliant parts is a challenging task in manufacturing automation. The critical issue is the deformations of the parts during handling, which is affected by both the end-effector design and the motion planning. This paper focuses on improving the productivity of multi-robot material handling systems by designing the structure of the end-effectors co-adaptively with the motion planning for the robot paths and trajectories. A novel multi-disciplinary methodology that enables this coadaptive simultaneous optimisation is proposed. The formulation for modelling this multi-disciplinary optimisation problem is presented, together with a detailed description of the proposed methodology's procedure.

The objectives of the methodology are to find the shortest cycle-time and smallest part deformations. The optimisation variables are the robot paths, trajectories, and end-effector designs. The constraints for the optimisation are to avoid parts being dropped, plastic part deformations, and collisions. The dynamic part deformations are considered during the collision detection simulation. Finally, a multi-robot coordination method determines the minimal relative starting time-delays for each operation to avoid collisions in the shared workspace and thereby also the shortest cycle-time.

The proposed methodology was evaluated for a realworld industrial case study that considers the multi-robot material handling system of a multi-stage tandem sheet metal press line. This demonstrates the need and contribution of the proposed methodology, i.e. that the parts' compliance must be considered and that substantial improvements in productivity can be achieved by co-adaptively optimising the end-effector designs and robot motion planning. The latter is the specific novelty of the proposed methodology compared to other exciting methods.

The unique element of this work is that the resulting end-effector designs are not only optimised for holding the handled parts but also for the collision-avoidance between the robots that are operating simultaneously in the shared workspace. This can have a significant influence on the productivity of the considered system, as demonstrated by conducted tests for the case study.

Future work could include a broader optimisation of the end-effector design, i.e. changing the number and type of vacuum-cups, and consider other mechanisms to hold parts such as clamps, fingers, shovels etc.

Acknowledgements The authors thank Nima K. Nia and Volvo Cars Corporation for their support and industrial insights concerning sheet metal press lines. This work was performed at the Digital LifeCycle Management (DLM) research group of WMG at University of Warwick in Coventry (UK), and supported by Västra Götalandsregionen (Sweden), under the grant PROSAM+ RUN 612-0208-16.
Open Access This article is distributed under the terms of the Creative Commons Attribution 4.0 International License (http:// creativecommons.org/licenses/by/4.0/), which permits unrestricted use, distribution, and reproduction in any medium, provided you give appropriate credit to the original author(s) and the source, provide a link to the Creative Commons license, and indicate if changes were made.

\section{References}

Bien Z, Lee J (1992) A minimum-time trajectory planning method for two robots. IEEE Trans Robot Autom 8(3):414-418

Binar Olofström AB (2014) Binar Press Automation UniFeeder. http://shop.binarolofstrom.se.k34.itc.se/index.php? id_product=2\&controller=product\&id_lang=1

Ceglarek D, Li HF, Tang Y (2001) Modeling and optimization of end effector layout for handling compliant sheet metal parts. ASME J Manuf Sci Eng 123(3):473-480

Datta R, Jain A, Bhattacharya B (2016) A piezoelectric model based multi-objective optimization of robot gripper design. Struct Multidisc Optim 53(3):453-470

Feng X, Patel R, Pons R, Casanelles R, Wappling D, Westrom J, Andersson H (2013) Optimization-based development of ultra high performance Twin Robot Xbar press tending robot system. In: 44th international symposium on robotics, pp 1-8

Franciosa P, Ceglarek D (2015) Hierarchical synthesis of multi-level design parameters in assembly system. CIRP Ann Manuf Technol 64(1):149-152

Franciosa P, Ceglarek D (2016) VRM Simulation toolkit. http:// www2.warwick.ac.uk/fac/sci/wmg/research/manufacturing/downloads/

Franciosa P, Das A, Ceglarek D, Bolognese L, Marine C, Mistry A (2014) Design synthesis methodology for dimensional management of assembly process with compliant non-ideal parts. In: Proceedings of joint conference on mechanical, design engineering \& advanced manufacturing

Franciosa P, Gerbino S, Ceglarek D (2016) Fixture capability optimisation for early-stage design of assembly system with compliant parts using nested polynomial chaos expansion. Procedia CIRP 41:87-92

Glorieux E, Danielsson F, Svensson B, Lennartson B (2015a) Constructive cooperative coevolutionary optimisation for interacting production stations. Int J Adv Manuf Technol 80(1-4):673-688

Glorieux E, Svensson B, Danielsson F, Lennartson B (2015b) Improved constructive cooperative coevolutionary differential evolution for large-scale optimisation. In: IEEE symposium on differential evolution, pp 3719-3726

Glorieux E, Svensson B, Danielsson F, Lennartson B (2015c) Simulation-based time and jerk optimisation for robotic press tending. In: 29th European simulation and modelling conference, EUROSIS, pp 377-384

Glorieux E, Svensson B, Danielsson F, Lennartson B (2016) Multiobjective constructive cooperative coevolutionary optimization of robotic press-line tending. Eng Optim 0(0):1-19

Hoffmann H, Kohnhäuser M (2002) Strategies to optimize the part transport in crossbar transfer presses. CIRP Ann Manuf Technol 51(1):27-32

Jansson T, Nilsson L, Redhe M (2003) Using surrogate models and response surfaces in structural optimization - with application to crashworthiness design and sheet metal forming. Struct Multidisc Optim 25(2):129-140

Jiménez P (2012) Survey on model-based manipulation planning of deformable objects. Robot Comput Integr Manuf 28(2):154163 
Kavraki LE, LaValle SM (2008) Motion planning. In: Siciliano B, Khatib O (eds) Springer handbook of robotics. Springer, Berlin, pp 109-131

Lambe AB, Martins JRRA (2012) Extensions to the design structure matrix for the description of multidisciplinary design, analysis, and optimization processes. Struct Multidisc Optim 46(2):273-284

Larsen E, Gottschalk SC, Lin M, Manocha D (2000) Fast proximity queries with swept sphere volumes. In: Proceedings of the IEEE international conference on robotics, pp 3719-3726

Li H, Ceglarek D (2002) Optimal trajectory planning for material handling of compliant sheet metal parts. ASME J Mech Des 124(2):213-222

Li HF, Ceglarek D, Shi J (2002) A dexterous part-holding model for handling compliant sheet metal parts. ASME J Manuf Sci Eng 124(1):109-118

Liao X, Wang GG (2003) Evolutionary path planning for robot assisted part handling in sheet metal bending. Robot Comput Integr Manuf 19(5):425-430

Mantriota G, Messina A (2011) Theoretical and experimental study of the performance of flat suction cups in the presence of tangential loads. Mech Mach Theory 46(5):607-617

Martins JR, Lambe AB (2013) Multidisciplinary design optimization: a survey of architectures. AIAA J 51(9):2049-2075

Patel R, Pons R, Casanelles R, Westrom J, Andersson H, Wappling D, Feng X (2013) Model driven mechatronic design optimization of a linear axis press tending robot. In: 44th international symposium on robotics, pp 1-4
Pettersson M, Ölvander J, Andersson H (2007) Application adapted performance optimization for industrial robots. In: IEEE international symposium on industrial electronics, pp 20472052

Platzer M, Feng X, Wappling D, Baumli N, Patel R, Pons R, Casanelles R, Westrom J (2013) New robot concept for ultra high performance press tending robot system. In: 44th international symposium on robotics, pp 1-3

Schmalz J, Giering L, Hölzle M, Huber N, Reinhart G (2016) Method for the automated dimensioning of gripper systems. Procedia CIRP 44:239-244

Shan S, Wang G (2010) Survey of modeling and optimization strategies to solve high-dimensional design problems with computationally-expensive black-box functions. Struct Multidisc Optim 41:219-241. 2

da Silva AM (2015) Mechatronics design process with energy optimization for industrial machines doctoral thesis. Marquette University, Wisconsin

Tuleja P, Sidlovská L (2014) Unilateral gripping with active vacuum suction cup: calculation of gripping force and number of suction cups. Tech Rep.: Transfer inovácií 29/2014. Technical University of Kosice, Slovakia

Wang H, Fan T, Li G (2017) Reanalysis-based space mapping metho, an alternative optimization way for expensive simulation-based problems. Struct Multidisc Optim 55:2143-2157

Zienkiewicz OC, Taylor RL (2000) The finite element method: basics v. 1, 5th edn. Butterworth-Heinemann Ltd, Oxford 